\title{
LA ALIANZA DEL PACÍFICO Y SU RELACIÓN CON EL EMPRENDIMIENTO INTERNACIONAL
}

César Antonio Monterroso Coronado*
Universidad Católica Sedes Sapientiae
amonterroso@ucss.edu.pe

Fecha de recepción: agosto de 2016

Fecha de aceptación: diciembre de 2016

ReSUMEN: En la presente investigación se detallará la relación de la actividad emprendedora internacional y el Acuerdo Comercial de la Alianza del Pacífico (AP) puesto en vigencia en el 2011. Asimismo, mediante la aplicación de un pool data a partir de fuentes oficiales del Proyecto Global Entrepreunership Monitor (GEM), World Competitiveness Center, Cepalstat, Doing Business, Index of Economic Freedom (IEF) y Foro Económico Mundial (FEM/WEF), se definirán los factores ambientales, subjetivos y objetivos

* César Antonio Monterroso Coronado es docente asociado de la Universidad Católica Sedes Sapientiae. Es magíster en Finanzas por la Universidad del Pacífico, MBA por la Universitá degli Studi di Genova y magíster en Administración de Negocios Internacionales por la UCSS. Actualmente desempeña el cargo académico de Coordinador de Economía Intermedia en la Facultad de Ciencias Económicas y Comerciales de la Universidad Católica Sedes Sapientiae. 
que pueden servir para la formación de políticas públicas de promoción de la actividad emprendedora internacional. Para lograrlo, el artículo presentará la metodología cuantitativa que pone en medición las relaciones teóricas y conceptuales propuestas en el modelo teórico-relacional de los factores ambientales, subjetivos y objetivos que articulan los efectos del Acuerdo Comercial de la AP sobre la actividad emprendedora internacional. Por último, los principales resultados de la investigación considerarán la relevancia estadística de los factores ambientales, subjetivos y objetivos en la promoción de la actividad emprendedora internacional dentro de la conformación de las políticas públicas propias del marco del Acuerdo Comercial de la AP. De igual forma, se presentarán recomendaciones a ser tomadas en cuenta en los grupos de trabajo técnico de la AP.

Palabras Clave: Actividad Emprendedora Internacional, Competitividad, Apertura Económica, Gasto de Infraestructura, Innovación, Mercado Internacional.

\section{THE PACIFIC ALLIANCE AND ITS RELATIONSHIP WITH INTERNATIONAL ENTREPRENEURSHIP ACTIVITY}

ABSTRACT: This research will detail the relationship between the international entrepreneurial activity and the Pacific Alliance (AP) Trade Agreement put into effect in 2011. Also, by applying a pool data from official sources of the Global Entrepreunership Project (GEF), World Competitiveness Center, Cepalstat, Doing Business, Index of Economic Freedom (IEF) and World Economic Forum (FEM / WEF) will define the environmental, subjective and objective factors that can be used for the formation of public policies of promotion of international entrepreneurial activity. To achieve this, the article will present the quantitative methodology that puts into measure the 
theoretical and conceptual relations proposed in the theoretical-relational model of environmental, subjective and objective factors that articulate the effects of the AP Trade Agreement on international entrepreneurial activity. Finally, the main results of the research will consider the statistical relevance of environmental, subjective and objective factors in the promotion of international entrepreneurial activity within the shaping of public policies within the framework of the PA Trade Agreement. Likewise, recommendations will be presented to be taken into account in the technical working groups of the PA.

KeYwords: International Entrepreneurship Activity, Competitiveness, Economic Opening, Infrastructure Spending, Innovation, International Market.

\section{Introducción}

T a Alianza del Pacífico (AP) es un mecanismo de integración abierta que, desde su aparición en el 2011, constituye un proceso innovador

en su temática y mecanismos de regulación de los Estados parte. Por un lado, se caracteriza por su afán de lograr la integración no solo de carácter económico sino además de político, social y cultural. Por el otro, ha funcionado como mecanismo de libre circulación de bienes, servicios, capitales y personas.

En el presente artículo se abordará la relación entre el nivel de actividad emprendedora internacional y la profundización de la AP, tanto en el plano comercial, de apoyo al sector empresarial, como en las herramientas institucionales implementadas para este fin. Con ello, se buscará entender los factores ambientales, subjetivos y objetivos que determinan el nivel de actividad emprendedora de carácter internacional, el cual puede alcanzarse 
dentro de los efectos del Acuerdo Marco y el Protocolo Adicional de la AP. Ello podría permitir al gestor de políticas públicas tener presente dichos factores en el grado de importancia al momento del diseño de un entorno proclive para el beneficio de la actividad emprendedora internacional.

\section{Marco Metodológico}

Teniendo como soporte la información disponible del Proyecto Global Entrepreunership Monitor $(\mathrm{GEM})^{1}$, World Competitiveness Center $^{2}$, Cepalstat ${ }^{3}$, Doing Business ${ }^{4}$, Index of Economic Freedom (IEF) ${ }^{5}$ y Foro Económico Mundial (FEM/WEF) ${ }^{6}$, para efectos del presente estudio, la base de datos se ha compuesto de un panel de datos para el periodo del 2007 al 2014. En cuanto a la información recopilada de las fuentes mencionadas, las variables en estudio se presentarán, a continuación, en la Tabla 1 .

1 Se encuentra disponible en http://www.gemconsortium.org/

2 Se encuentra disponible en https://www.imd.org/wcc/world-competitiveness-center/ 3 Se encuentra disponible en http://estadisticas.cepal.org/

4 Se encuentra disponible en espanol.doingbusiness.org/

5 Se encuentra disponible en http://www.heritage.org/index/

6 Se encuentra disponible en http://reports.weforum.org/global-competitivenessindex/downloads/ 


\section{Tabla 1}

Matriz de Variables

\begin{tabular}{|c|c|c|c|}
\hline Variable & Descripción & Tratamiento & Fuente \\
\hline TEAYYINT & $\begin{array}{l}\text { Tasa de actividad } \\
\text { emprendedora }\end{array}$ & Variable cuantitativa en $\%$ & GEM APS \\
\hline VC4 & $\begin{array}{l}\text { Intercambio comercial de } \\
\text { Estados Parte de la AP }\end{array}$ & $\begin{array}{l}\text { Medida en tasa de crecimiento } \\
\text { porcentual }\end{array}$ & CepalStat \\
\hline IC & Índice de competitividad & Valor del índice & WEF \\
\hline GI & Gasto en infraestructura & $\begin{array}{l}\text { Medida en tasa de crecimiento } \\
\text { porcentual }\end{array}$ & CepalStat \\
\hline EOSQ068 & $\begin{array}{l}\text { Indicador de absorción } \\
\text { tecnológica a nivel } \\
\text { empresarial [1- } 7 \text { (el mejor)] }\end{array}$ & $\begin{array}{l}\text { Variable cualitativa politómica. } \\
\text { Escala de Likert }\end{array}$ & WEF \\
\hline FGNMKTIDX & $\begin{array}{l}\text { Índice de tamaño del } \\
\text { mercado extranjero [1- } 7 \text { (el } \\
\text { mejor)] }\end{array}$ & $\begin{array}{l}\text { Variable cualitativa politómica. } \\
\text { Escala de Likert }\end{array}$ & WEF \\
\hline OPPORTYY & Percepción de oportunidades & $\begin{array}{l}\text { Variable cualitativa politómica. } \\
\text { Escala de Likert }\end{array}$ & GEM APS \\
\hline KNA11IMD & Igualdad de oportunidades & $\begin{array}{l}\text { Variable cualitativa politómica. } \\
\text { Escala de Likert }\end{array}$ & $\begin{array}{l}\text { IMD World } \\
\text { Competitiveness } \\
\text { Center }\end{array}$ \\
\hline EOSQ118 & $\begin{array}{l}\text { Gasto en } \mathrm{I}+\mathrm{D} \text { de la empresa } \\
{[1-7 \text { (el mejor) }]}\end{array}$ & $\begin{array}{l}\text { Variable cualitativa politómica. } \\
\text { Escala de Likert }\end{array}$ & WEF \\
\hline KNTCE & $\begin{array}{l}\text { Costo para exportar (dólares } \\
\text { por conteiner) }\end{array}$ & $\begin{array}{l}\text { En unidades monetarias } \\
\text { extranjeras }\end{array}$ & Doing Business \\
\hline KNSBC & $\begin{array}{l}\text { Costo de iniciar un negocio } \\
\text { (\% del ingreso per cápita) }\end{array}$ & $\begin{array}{l}\text { Como tasa porcentual de } \\
\text { participación en el ingreso per } \\
\text { cápita }\end{array}$ & Doing Business \\
\hline EOSQ353 & $\begin{array}{l}\text { Calidad de la infraestructura } \\
\text { portuaria [1- } 7 \text { (el mejor)] }\end{array}$ & $\begin{array}{l}\text { Variable cualitativa politómica. } \\
\text { Escala de Likert }\end{array}$ & WEF \\
\hline EOSQ104 & $\begin{array}{l}\text { Efectividad de la política } \\
\text { antimonopolio [1-7 (el } \\
\text { mejor)] }\end{array}$ & $\begin{array}{l}\text { Variable cualitativa politómica. } \\
\text { Escala de Likert }\end{array}$ & WEF \\
\hline KNTF & Índice de comercio libre & $\begin{array}{l}\text { Valor numérico del índice según } \\
\text { la metodología IEF }\end{array}$ & $\begin{array}{l}\text { Index of Economic } \\
\text { Freedom }\end{array}$ \\
\hline
\end{tabular}


Para efectos de tener un control sobre las heterogeneidades estructurales y económicas de los Estados parte, se aplicó una regresión de pool de datos en el Programa Eviews versión 9.5. En esta técnica econométrica se puede controlar de forma simultánea la heterocedasticidad y autocorrelación presente como producto de la diversidad económica y social de los Estados parte firmantes del Acuerdo Marco y Protocolo Adicional del Acuerdo Comercial de la AP.

\section{Marco Teórico}

En la raíz de la investigación sobre el tema de emprendimiento se encuentra una serie de teorías asociadas al desarrollo de esta actividad emprendedora. Por un lado, las teorías institucionales, que plantean las relaciones o instituciones establecidas dentro del marco de una serie de acuerdos comerciales, que permiten el crecimiento sostenible de esta actividad. Por otro lado, las teorías psicológicas asociadas al carácter del emprendedor, su experiencia y motivación para emprender, así como las teorías de la complejidad sistémica, entendiendo el emprendimiento como una actividad tanto individual como colectiva. En este sentido, las variables del entorno social son fundamentales para su comprensión.

En la Figura 1, el modelo teórico-relacional presentará los factores intervinientes e influyentes de la actividad emprendedora internacional. Por un lado, los factores ambientales, los cuales establecen las condiciones sobre la cual se desenvuelve y desarrolla esta actividad. Por otro lado, la actividad emprendedora internacional condicionada puede ser explicada por factores subjetivos y objetivos, tanto a nivel del emprendedor y su percepción como macroeconómico de la actividad dentro del contexto del Acuerdo Comercial de la AP. 
Factores subjetivos

1. Microeconómicos OPPORTYY

KNA11IMD

2.Macroeconómicos

IC

EOSQ104

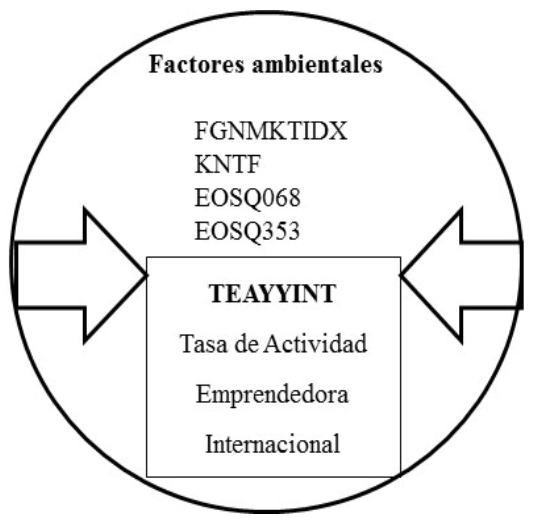

Factores objetivos

1. Microeconómicos

KNSBC

KNA11IMD

2.Macroeconómicos

GI

$\mathrm{VC} 4$

EOSQ118

KNTCE

Figura 1. Modelo teórico-relacional.

\section{Marco Conceptual}

Para efectos de la presente investigación, se ha empleado un determinado conjunto de categorías conceptuales. Estas abarcarán los aspectos a ser medibles con el fin de lograr un contraste de la hipótesis central acerca de la eficacia de la formación del Acuerdo Comercial de la AP para incrementar la tasa de actividad emprendedora en la región. A continuación, se detallarán dichas categorías.

\subsection{Tasa de actividad emprendedora internacional (TEAYYINT)}

En el GEM se mide la tasa de actividad emprendedora como el porcentaje de individuos adultos entre 18 y 64 años. Los mismos se hallan inmersos en el proceso empresarial de la puesta en marcha de un negocio o empresa, del cual pueden poseer parte o todo el activo operativo. De igual 
forma, pueden ser o ser ya propietarios-gerentes de una empresa joven, la cual también incluya el autoempleo.

Para su medición, el GEM incorpora la metodología desarrollada por Reynolds, Bosma, Autio, Hunt, De Bono y Servias (2005). Esta serie de indicadores se detallarán a continuación:

- El porcentaje de emprendedores potenciales medido por el número de personas de la población adulta que contestaron afirmativamente a la intención de poner en marcha un negocio o empresa en los próximos tres ańos.

- El porcentaje de emprendedores nacientes medido por el número de personas adultas que poseen y gestionan un negocio o empresa que no les permite generar salarios o ingresos por más de tres meses. Estos negocios o empresas aún no se han consolidado o establecido económicamente.

- El porcentaje de emprendedores nuevos medido por el número de personas adultas que poseen un negocio o empresa que les permite generar salarios o ingresos entre 3-42 meses, o hasta 3.5 años. Estos negocios o empresas aún no se han consolidado o establecido económicamente.

- La tasa de actividad emprendedora (TEAYYINT) mide el nivel de actividad emprendedora en la etapa inicial de la formación de nuevos negocios o empresas. Es el indicador más importante del Proyecto GEM. 
- El porcentaje de empresarios consolidados que son aquellos que gestionan un negocio o empresa que ha logrado hacer pago de salarios por más de 42 meses (3.5 años).

\section{2 Índice de competitividad (IC)}

El índice de competitividad calculado por el Foro Económico Mundial (FEM) se entiende como un conjunto de instituciones, medidas de políticas y factores que determinan el nivel de productividad de una economía generando su prosperidad. Mediante estas, la actividad emprendedora puede servirse para incrementar la productividad de su actividad en el largo plazo. Este indicador incorpora 114 indicadores de organizaciones internacionales entre los que figuran el Fondo Monetario Internacional (FMI), el Banco Mundial (BM) y varias agencias de la Naciones Unidas (ONU), incluyendo la UNESCO y la Organización Mundial de la Salud (OMS). Asimismo, la encuesta de opinión ejecutiva del FEM, que refleja los aspectos cualitativos de la competitividad, se compone de 12 pilares agrupados en tres niveles de medición (Schwab, 2016):

- Requerimientos básicos: incorpora cuatro pilares sobre los cuales mínimamente se sostiene la competitividad de un país, e impulsan a estos al logro de este objetivo en el largo plazo (factordriven economies). Estos se mencionarán a continuación: (a) Instituciones: estas expresan el marco jurídico y administrativo, la calidad de las instituciones públicas; (b) Infraestructura: incluye los modos de transporte, el movimiento de los trabajadores hacia empleos adecuados y la red de telecomunicaciones; (c) Entorno macroeconómico: incluye el balance del presupuesto público, 
ahorro nacional bruto, tasa anual de inflación, deuda pública, ranking crediticio del país; y (d) Educación primaria y Salud: calidad de la educación primaria, gasto neto en educación primaria, impacto de la malaria, tuberculosis, HIV/AIDS en los negocios, la mortalidad infantil y la esperanza de vida en años.

- Requerimientos potenciadores de eficiencia: incorpora seis pilares que impulsan a los países hacia el logro de la eficiencia como objetivo de largo plazo (efficiency-driven economies). Estos son los que siguen: (a) Entrenamiento y Educación superior: incluye encuesta sobre la educación secundaria y terciaria, calidad del sistema educativo, calidad de la educación en ciencias y matemáticas, acceso de internet en las escuelas, disponibilidad de servicios de entrenamiento e investigación y entrenamiento en el trabajo; (b) Eficiencia del mercado de bienes: incluye intensidad de la competencia local, extensión del dominio del mercado, efectividad de la política antimonopolio, efecto de la tasa impositiva sobre la motivación a invertir, número de procedimientos para iniciar un negocio, número de días para iniciar un negocio, costos de la política agrícola, tasa impositiva total, prevalencia de las barreras comerciales, prevalencia de la propiedad extranjera, impacto sobre los negocios de la normativa sobre la inversión extranjera directa, costos de los procedimientos aduaneros, aranceles, grado de orientación al cliente, sofisticación del comprador y calidad de las condiciones de la demanda; (c) Eficiencia del mercado de trabajo: incluye cooperación entre empleado y empleador, prácticas de contratación y despidos, fijación flexible del salario, efectos de la fiscalización a la generación de empleo, pagos y productividad, 
capacidad del país para retener y atraer el talento, ratio de feminidad en el empleo y eficiencia en el uso del talento; (d) Desarrollo del mercado financiero: incluye el acceso a los servicios financieros, servicios financieros que satisfacen las necesidades empresariales, financiación a través del mercado local de renta variable, facilidad de acceso al préstamo bancario, disponibilidad de capital de riesgo, solidez de los bancos, regulación del intercambio de valores e índice de derechos legales; (e) Preparación tecnológica: incluye la disponibilidad de nuevas tecnologías, absorción de tecnología a nivel de la empresa, inversión extranjera directa y transferencia tecnológica, individuos utilizando internet, suscripción a internet de banda ancha fija, ancho de banda de internet por usuario, suscripciones de banda de ancho móvil; y (f) Tamaño del mercado: incluye al PBI (PPP \$ miles de millones), exportaciones como porcentaje del PBI, índice de tamaño del mercado interno e índice de tamańo del mercado externo.

- Requerimiento potenciador de innovación y sofisticación: incorpora dos pilares que impulsan a las economías al logro de la innovación en el largo plazo (innovation-driven economies). Estos se detallarán a continuación: (a) Sofisticación de los negocios: incluye la cantidad de proveedores locales, estado de desarrollo del clúster, naturaleza de la ventaja competitiva, sofisticación del proceso productivo, voluntad para delegar la autoridad, control de la distribución internacional, extensión de la comercialización y (b) Innovación: incluye a la capacidad de innovación, calidad de las instituciones de investigación científica, gasto de la empresa $\mathrm{I}+\mathrm{D}$, colaboración universitaria-industria en $\mathrm{I}+\mathrm{D}$, adquisición de productos 
tecnológicos avanzados por parte del gobierno, disponibilidad de científicos e ingenieros y aplicaciones de patentes (PCT) sobre el millón de personas.

\section{3 Índice de apertura económica (VC4)}

El índice de apertura económica expresa el grado de participación de la economía local en el mercado mundial de bienes y servicios. Igualmente, permite caracterizar a una economía nacional como de mayor sensibilidad al mercado externo. Esto implica que los acuerdos o tratados de esta economía le permiten a la actividad emprendedora local, con capacidades para enfrentar la globalización, incrementar sus operaciones o negocios comerciales (Bachetta, Beverelli, Cadot, Fugazza, Grether, Heible, Nicita \& Piermartini, 2012). En el contexto de la presente investigación, se interpretará cómo la tasa de crecimiento de la apertura económica, producto del Acuerdo Comercial de la AP, en función de la integración económica de los Estados parte, medida por el volumen comercial dispuesta al mercado internacional vía los acuerdos de libre comercio implementados dentro del Marco del Acuerdo Comercial que muestra la relación esperada entre la apertura económica y la actividad emprendedora internacional.

\subsection{Indicador de gasto en infraestructura (GI, EOSQ353)}

En los estudios de la actividad emprendedora se muestra la importancia de la infraestructura asociada a las exportaciones. En tal sentido, el efecto positivo esperado de la magnitud de gasto de infraestructura (GI), en la formación de la actividad emprendedora de carácter internacional, se evidencia por los efectos impulsores de la infraestructura asociada a la 
actividad exportadora. Por su parte, el indicador EOSQ353 de calidad de la infraestructura portuaria, que habilita el desarrollo exportador de la actividad emprendedora de carácter internacional, se vuelve la base logística para su desarrollo (Molina, Heuser, \& Mesquita Moreira, 2016).

\subsection{Indicadores de innovación (EOSQ068, EOSQ118)}

El indicador EOSQ068 representa el grado de absorción de tecnología a nivel empresarial. De igual forma, el indicador EOSQ118 refiere el gasto en investigación y desarrollo de la empresa que realiza la actividad emprendedora de carácter internacional. Estos indicadores permiten observar la importancia de los procesos y estrategias de innovación dentro del Acuerdo Marco y Protocolo Adicional de la AP (García-Pérez de Lema, Gálvez-Albarracín, \& Maldonado-Guzmán, 2016).

\subsection{Indicadores de mercado internacional (FGNMKTIDX, KNTF, EOSQ104, KNSBC, OPPORTYY, KNA11IMD)}

Para el desarrollo de la actividad emprendedora internacional, y su proceso de internacionalización, se requiere medir los efectos del mercado internacional sobre la puesta en marcha del Acuerdo Comercial de la AP (Hors, 2015). En tal sentido, indicadores como el índice sobre el mercado internacional (FGNMKTIDX), que representa cuál es la importancia del mercado destino, y el índice de comercio libre (KNTF), una de las limitaciones de la actividad emprendedora en los mercados externos, se asocian a la efectividad de la ley de antimonopolio (EOSQ104). Es importante, también, reconocer el porcentaje del ingreso per cápita del emprendedor asociado a las exportaciones como costo administrativo para 
iniciar un negocio internacional (KNSBC), así como aspectos reglamentarios de igualdad de oportunidades que ofrece el mercado destino para la actividad emprendedora internacional (KNA1 1IMD) y la percepción de oportunidad de negocios que ofrece el mercado destino para la actividad emprendedora internacional (OPPORTYY).

\section{Desarrollo y Análisis}

Para esta sección, se ha partido de una muestra de información económica de los Estados parte de la AP para el periodo del 2007 al 2014. Ahora bien, sobre las variables del presente estudio en relación con la actividad emprendedora internacional, se puede dejar en claro distintos términos que se presentarán a continuación.

\subsection{Comercio libre}

El comercio libre permite una apertura de mercados para la actividad emprendedora que no siempre es beneficiosa para toda economía. En el caso de Chile y Colombia, del 2007 al 2014, la relación no es estable en el sentido que en algunos años esta deja de guardar una relación directa estable. No obstante, en el caso de México y Perú, la relación positiva es estable, en el sentido que, a mayor índice de comercio libre, se espera un crecimiento de la actividad emprendedora. Dicha relación se ve expuesta a otras condiciones propias del mercado internacional que enfrenta la actividad emprendedora internacional. Por ende, cuando se observa dicha relación como un conjunto, vale decir, cuando las economías individuales en integración obtienen operaciones comerciales para la actividad emprendedora, pueden registrar efectos inversos a lo esperado. Los casos de los países mencionados se observarán la Figura 2. 


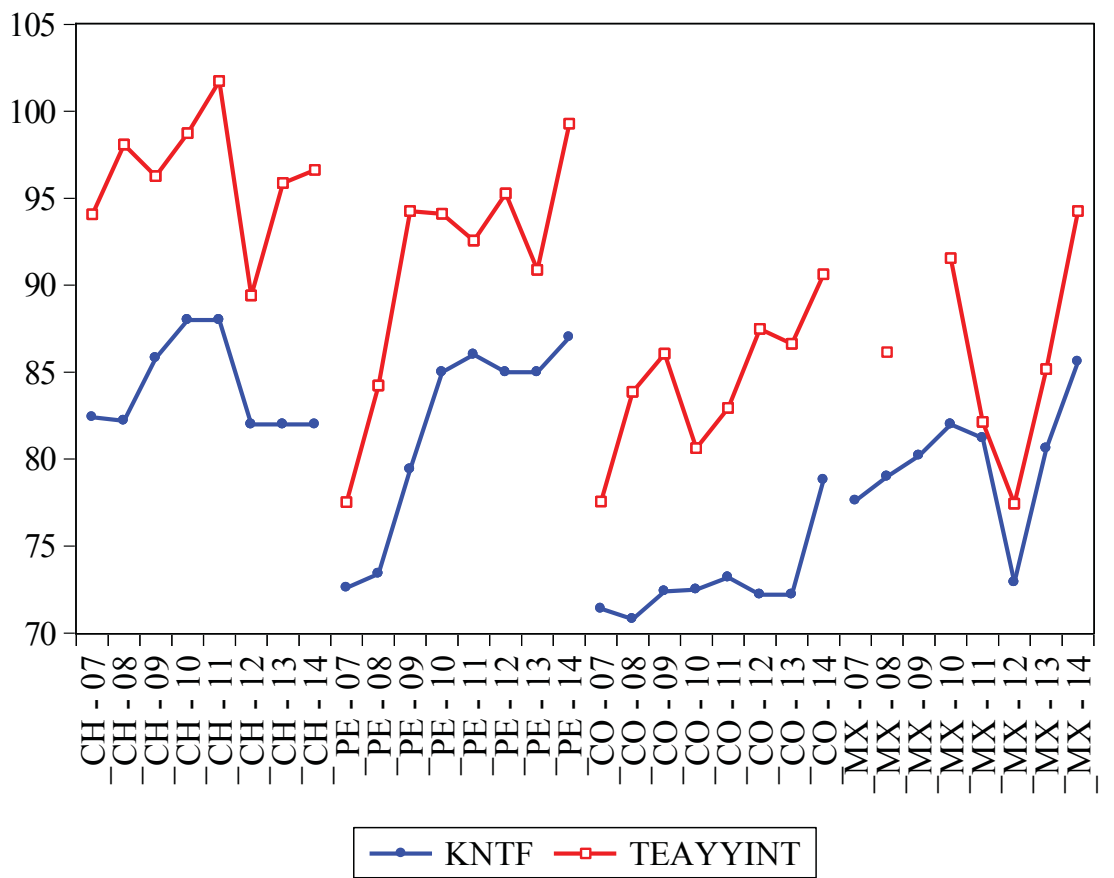

Figura 2. Comercio libre y actividad emprendedora internacional $(\mathrm{CH}=\mathrm{Chile}$, $\mathrm{PE}=$ Perú, $\mathrm{CO}=$ Colombia, $\mathrm{MX}=$ México).

\subsection{Calidad de puertos}

La calidad de puertos no asegura la relevancia de la actividad emprendedora internacional. Si bien mejora las condiciones del comercio internacional en general, empero, incluye actividades de exportación directa e indirecta que no necesariamente le dan posicionamiento en el mercado destino. Para países como México y Chile una mejora en la calidad de los puertos ha incrementado la actividad emprendedora de carácter internacional. En contraste, en los casos de Perú y Colombia, esta no ha 
significado necesariamente una mejora de la actividad emprendedora de carácter exportador, en tanto la actividad comercial de estos países es de naturaleza errática, y de acuerdo con el entorno macroeconómico externo, el cual se observará en la Figura 3.

En conjunto, los efectos de la calidad del servicio portuario para el Acuerdo de la AP han presentado una relación inversa a lo teóricamente esperado. Debido al peso de los efectos políticos sobre las economías de los Estados parte, ha generado un beta negativo de -9.13 con significancia del 1 $\%$. Estos resultados se detallarán en la Figura 3.

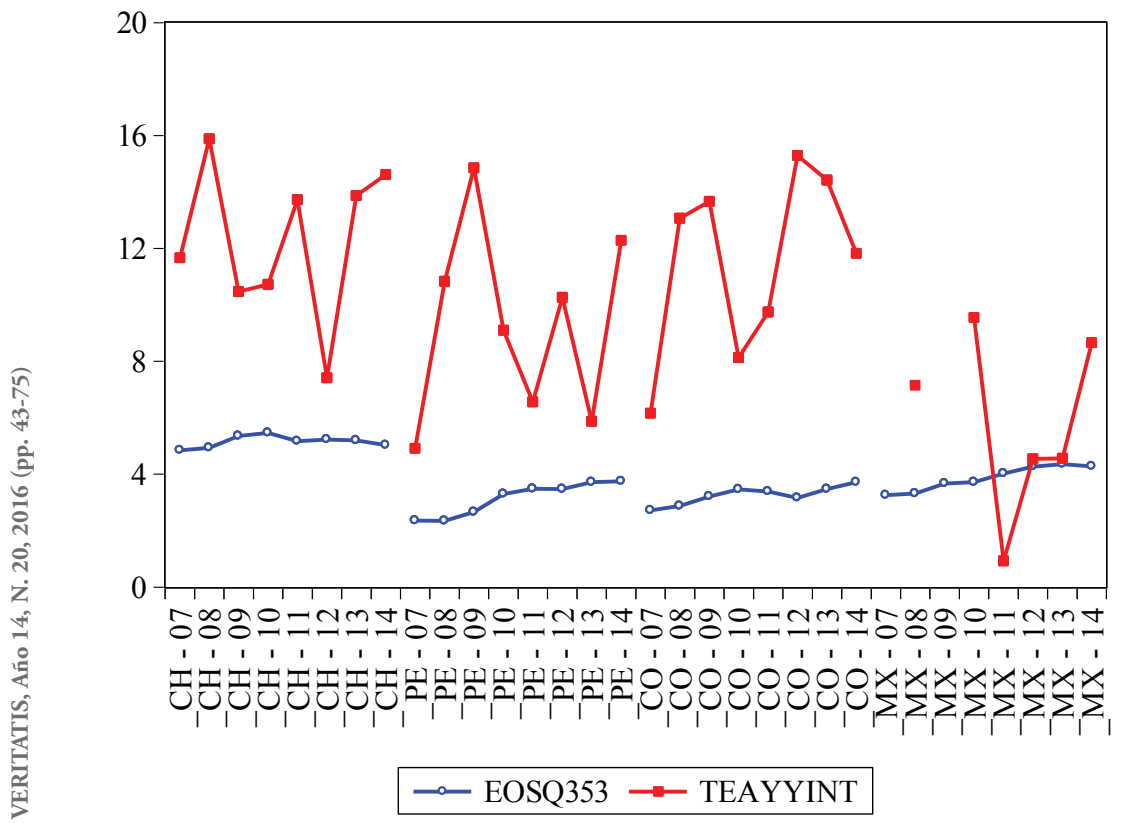

Figura 3. Calidad de puertos y actividad emprendedora internacional $(\mathrm{CH}=\mathrm{Chile}$, $\mathrm{PE}=$ Perú, $\mathrm{CO}=$ Colombia, $\mathrm{MX}=$ México). 


\subsection{Apertura económica}

La apertura económica medida como la tasa porcentual de la profundización del valor comercial entre los Estados parte, como consecuencia del Acuerdo Comercial de la AP, ha generado efectos positivos en la generación de la actividad emprendedora de carácter internacional. A pesar que la relevancia estadística del beta estimado de 3.65 al $1 \%$, tal como se presentará en la Tabla 2. En algunos años, como el 2008, la crisis internacional afectó las ventajas de la apertura económica como producto de la AP y consiguientemente su efecto positivo sobre la generación de actividad emprendedora internacional. En la Figura 4, este efecto negativo se manifestó de forma diferenciada entre Perú-Colombia y Chile-México. En tal sentido, la pérdida de operaciones comerciales de carácter internacional para Chile-México se ha visto más afectada que Perú-Colombia.

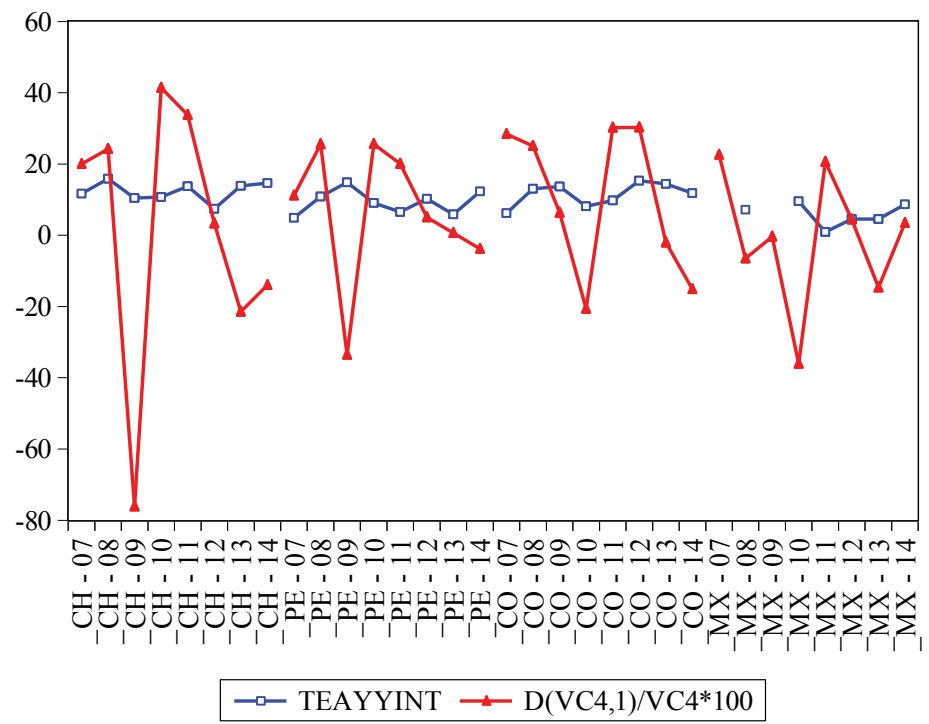

Figura 4. Apertura económica y actividad emprendedora internacional $(\mathrm{CH}=\mathrm{Chile}$, $\mathrm{PE}=$ Perú, $\mathrm{CO}=$ Colombia, MX=México). 


\subsection{Gasto en infraestructura}

La tasa de gasto en infraestructura física explica el proceso histórico de formación de una infraestructura física que permita el desarrollo de la actividad exportadora de carácter internacional. Esta variable ha tenido efectos cíclicos con retardos, debido a que sus efectos no se observan en el periodo inmediato sino en un año. En la Figura 5 se demostrará este resultado a través de un beta positivo de 3.51 al $1 \%$ de significancia.

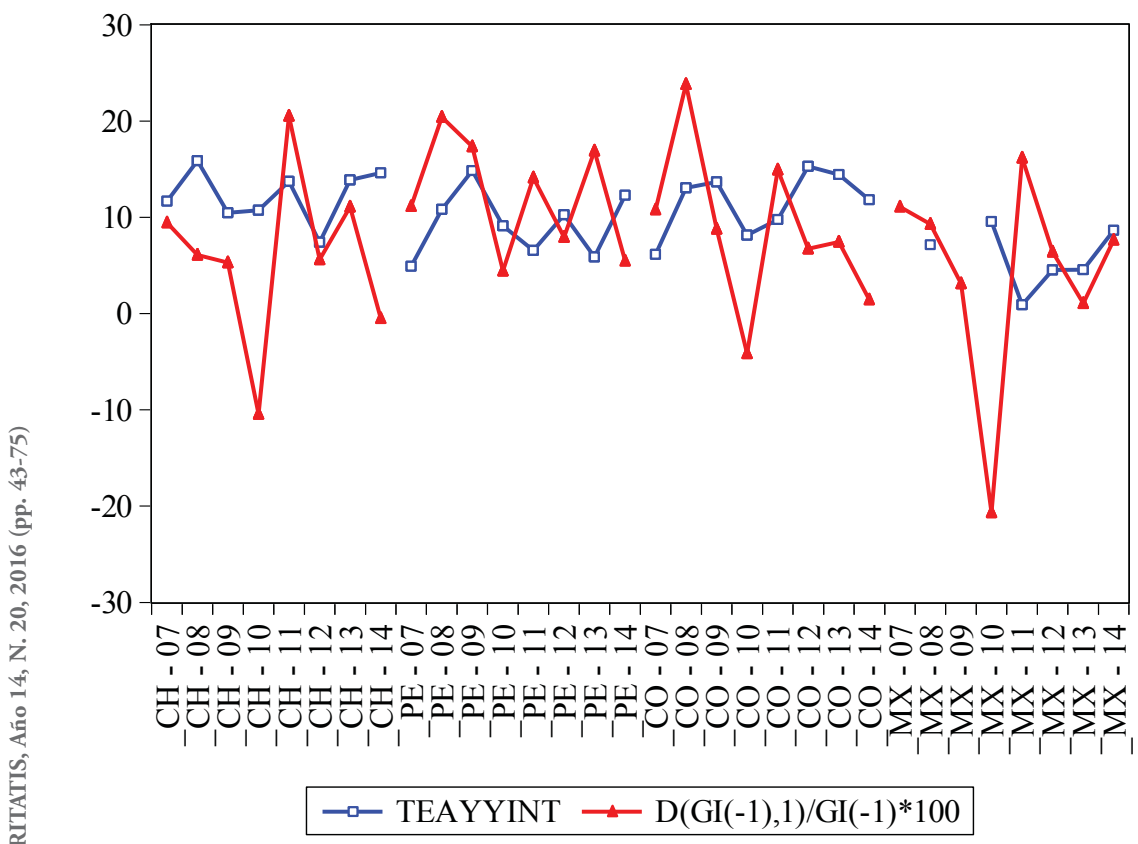

Figura 5. Gasto en infraestructura y actividad emprendedora internacional ( $\mathrm{CH}=$ Chile, $\mathrm{PE}=$ Perú, $\mathrm{CO}=$ Colombia, $\mathrm{MX}=$ México). 


\subsection{Competitividad}

La competitividad del país como indicador del WEF ha sido observada en el periodo de formación y consolidación del Acuerdo de la Alianza del Pacífico. Como se observará en la Figura 6, la calificación de la competitividad por cada país analizado se ha mantenido casi constante, con poca variación, siendo a pesar de ello positiva para efectos de explicar la actividad emprendedora internacional al $1 \%$ de significancia. Por otra parte, el efecto observado de aumento leve del nivel de competitividad, se ha presentado con un incremento de la actividad emprendedora internacional tal como se observará después, empíricamente, en la Tabla 2.

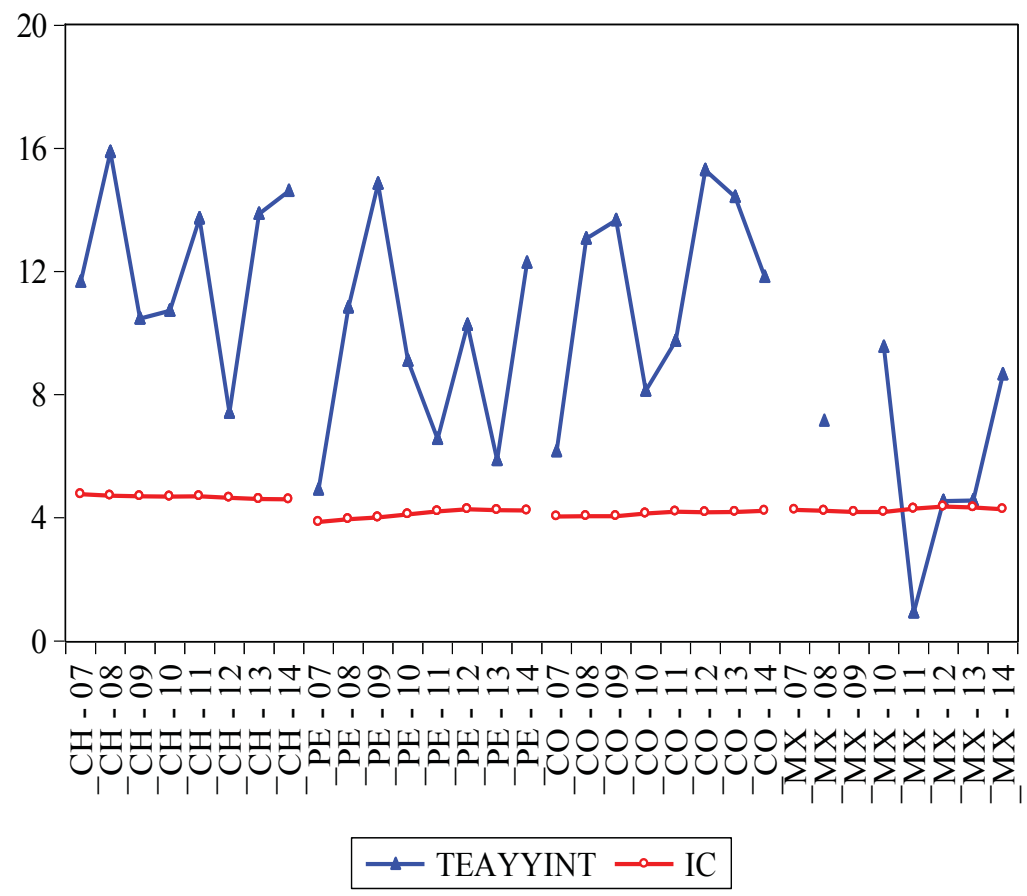

Figura 6. Competitividad y actividad emprendedora internacional $(\mathrm{CH}=\mathrm{Chile}$, $\mathrm{PE}=$ Perú, $\mathrm{CO}=$ Colombia, MX=México). 


\subsection{Absorción de tecnología}

La absorción de tecnología en la literatura de negocios internacionales se ve asociado con la competitividad empresarial de la actividad emprendedora internacional. De ese modo, la absorción o transferencia de tecnología en el proceso de internacionalización beneficia a Perú-Colombia frente a ChileMéxico. Esto se asocia a la actividad emprendedora internacional que se generaría por el aprendizaje de Perú-Colombia frente a Chile-México. Asimismo, tuvo como vía la regularización de las buenas prácticas comerciales y otros aportes de homogenización de la actividad emprendedora por la puesta en marcha del Protocolo Adicional en el 2011, durante el contexto del Acuerdo Comercial de la AP. A continuación, dichas precisiones se ejemplificarán en la Figura 7.

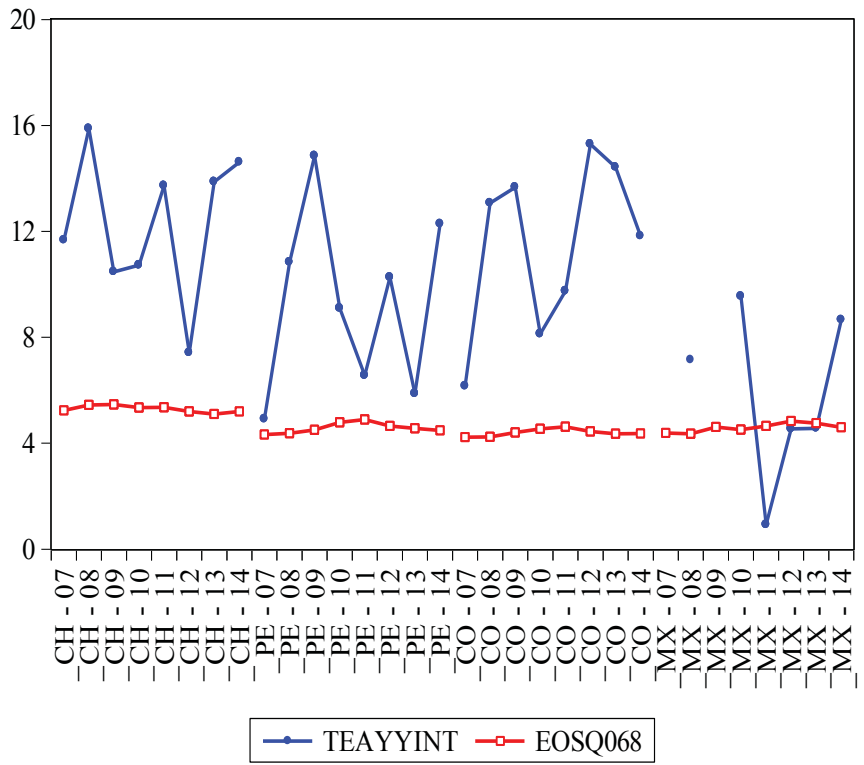

Figura 7. Absorción de tecnología y actividad emprendedora internacional ( $\mathrm{CH}=$ Chile, $\mathrm{PE}=$ Perú, $\mathrm{CO}=$ Colombia, $\mathrm{MX}=$ México). 


\subsection{Costo de iniciar un negocio}

El costo de iniciar un negocio es una barrera a la entrada de las operaciones en el mercado internacional. Un incremento del costo de iniciar un negocio significa reducir la capacidad de generar una nueva actividad emprendedora de carácter internacional. Para los Estados parte con información histórica registrada, como es el caso de Perú, Chile y Colombia, se presenta una relación inversa en el sentido esperado por la teoría de los negocios internacionales. Es decir, independientemente del nivel de desarrollo de la economía del Estado parte, una reducción efectiva del costo de apertura de negocios en el ámbito internacional puede generar beneficios en la actividad emprendedora internacional, diferenciados y asociados al grado de aprendizaje de las empresas exportadoras. En la Figura 8 se detallarán los datos mencionados.

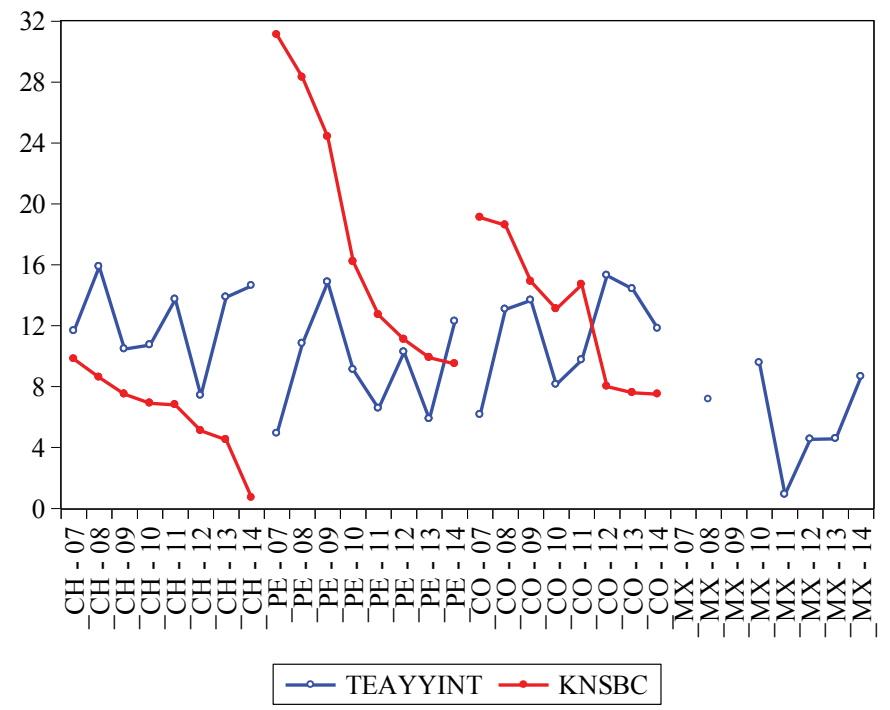

Figura 8. Costo de iniciar el negocio y actividad emprendedora internacional ( $\mathrm{CH}=$ Chile, $\mathrm{PE}=$ Perú, $\mathrm{CO}=$ Colombia, $\mathrm{MX}=$ México). 


\subsection{Gasto en investigación y desarrollo}

El gasto en investigación y desarrollo asociado a mejorar la operatividad de los negocios internacionales de la actividad emprendedora de carácter internacional, muestra una relación positiva o directa, lo cual se describirá propiamente en la Figura 9. De esa forma, a mayor desembolso del presupuesto financiero de las empresas en investigación para mejorar el proceso de hacer negocios, se tiene un indicador de 22.89 al $1 \%$ de significancia. Dicho resultado irá acorde con lo mostrado más adelante en la Tabla 2.

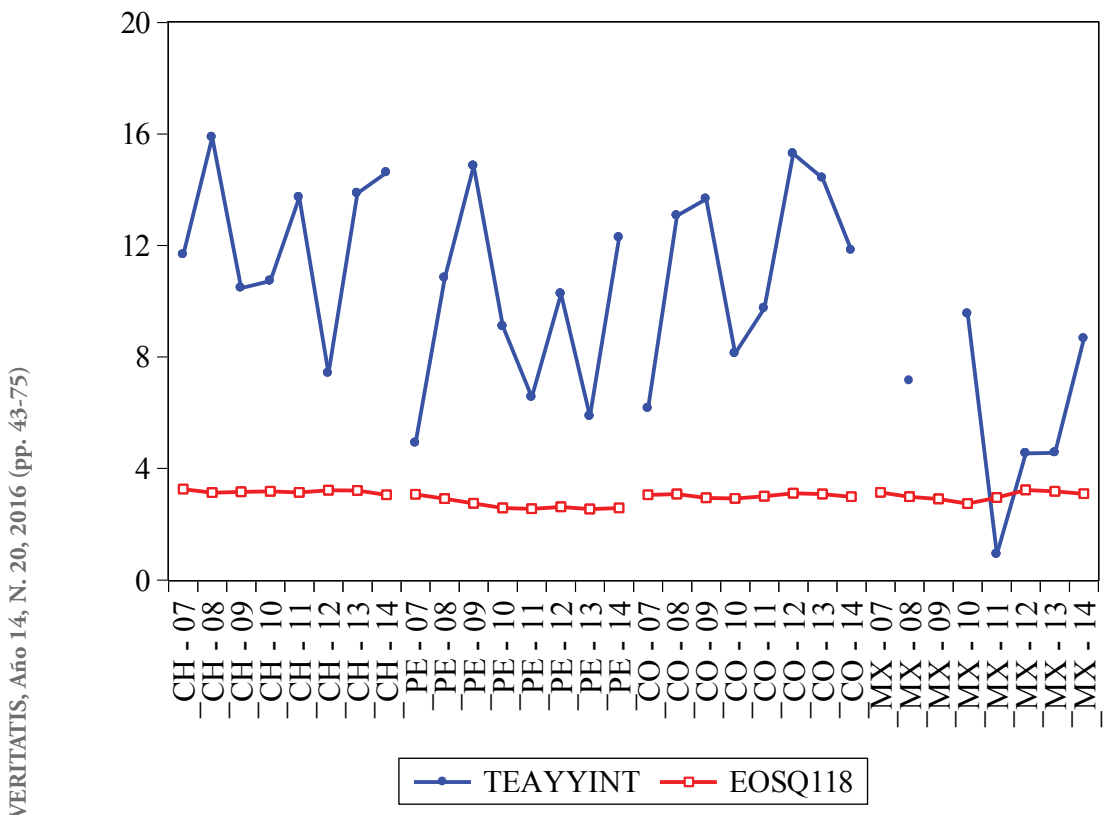

Figura 9. Gasto $\mathrm{I}+\mathrm{D}$ y actividad emprendedora internacional $(\mathrm{CH}=\mathrm{Chile}, \mathrm{PE}=$ Perú, $\mathrm{CO}=$ Colombia, $\mathrm{MX}=$ México). 


\subsection{Importancia del mercado internacional}

La importancia del mercado internacional como mercado destino tiene teóricamente una relación directa. Esto significa que, cuando un mercado destino es de importancia creciente para hacer negocios, se asocia con una mayor participación de la actividad emprendedora internacional asociada a un Estado parte. Esta relación se evidencia para Perú-Colombia, pero en el caso de México-Chile no se observa de forma estable como se verá en la Figura 10. Igualmente, cuando se mide la relación dentro del contexto del Acuerdo Comercial de la AP, el peso relativo de importancia de los Estados parte México-Chile en el Acuerdo Comercial determina una relación positiva que se ve reforzada por la participación de los Estados parte PerúColombia determinando un efecto de 14.34 al $1 \%$ de significancia. De ese modo, estaría indicando la integración económica mediante la modalidad de tratados de libre comercio homogéneos que se configuran en el Protocolo Adicional puesto en marcha desde el 2011. Así, permitiría el desarrollo de la actividad emprendedora de carácter internacional dependiendo de la importancia económica de los mercados destino. 


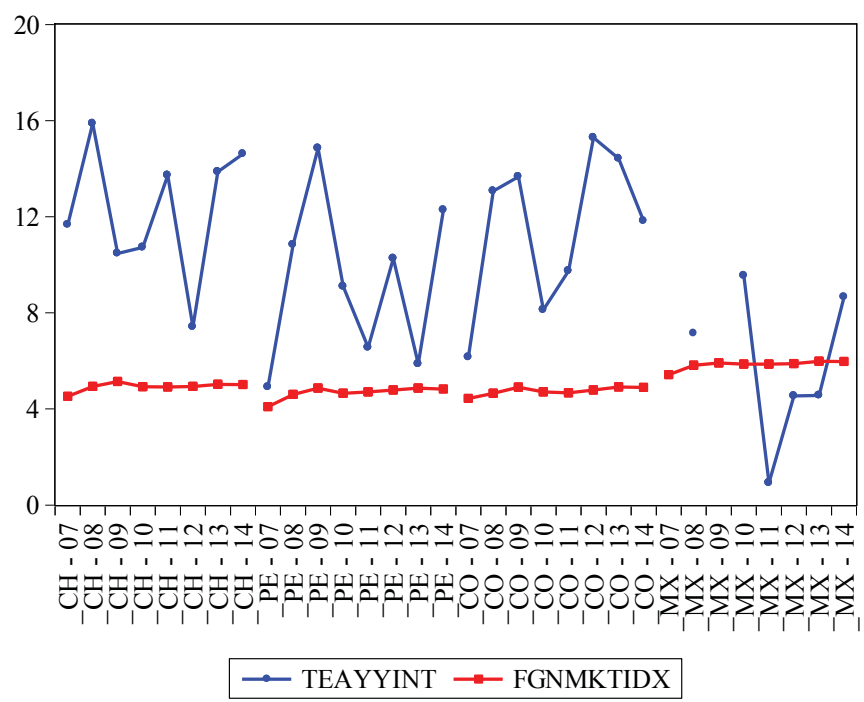

Figura 10. Importancia del mercado internacional y actividad emprendedora internacional ( $\mathrm{CH}=$ Chile, $\mathrm{PE}=$ Perú, $\mathrm{CO}=$ Colombia, MX=México).

\subsection{Igualdad de oportunidades}

La igualdad de oportunidades en los negocios, que constituye una institución en los negocios internacionales, implica, para el desarrollo de la actividad emprendedora de carácter internacional, la necesidad de una regulación o instituciones que permitan un incremento de la actividad emprendedora internacional. Para el caso de Perú-Colombia se expresa en una relación positiva, es decir, un ambiente igualitario del capital extranjero en el mercado destino, permite un mayor desarrollo de la actividad emprendedora. Pero, en el caso de México-Chile, lo que puede asociarse a la mayor diversificación de mercados destino por tratados de libre comercio que poseen estos países del Acuerdo Comercial de la AP, revela que los 
efectos de igualdad de trato comercial al capital extranjero puede diluirse o dejar de tener influencia por esta diversificación de mercados destinos; para efectos de empujar la actividad emprendedora de carácter internacional.

En conjunto, el efecto final del Acuerdo Comercial ha significado un beta negativo de 2.21 significativo estadísticamente al $1 \%$. En otras palabras, significa que la heterogeneidad económica de los Estados parte y su capacidad exportadora a través de la actividad emprendedora puede volverse irrelevante para efecto de los Estados parte de menor desarrollo. Con esto, ante menores oportunidades de trato igualitario, la actividad emprendedora internacional puede crecer para Perú-Colombia por la potencia del comercio que genera el Acuerdo Comercial de la AP. A continuación, la Figura 11 plasmará los datos mencionados.

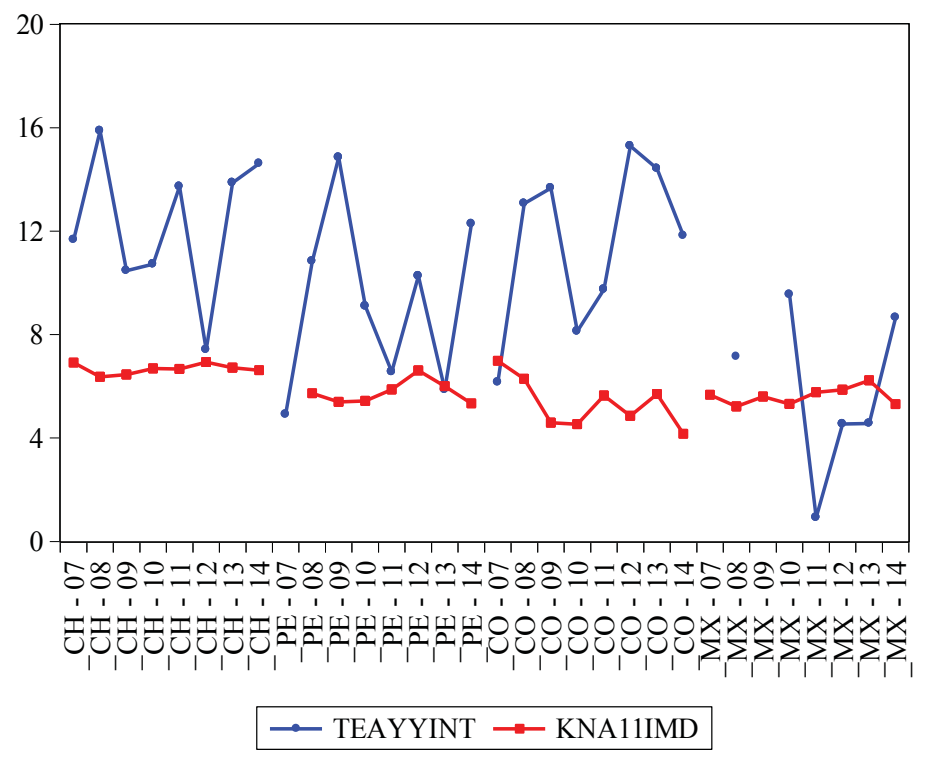

Figura 11. Igualdad de oportunidades y actividad emprendedora internacional ( $\mathrm{CH}=$ Chile, $\mathrm{PE}=$ Perú, $\mathrm{CO}=$ Colombia, $\mathrm{MX}=$ México). 


\subsection{Regulación antimonopolio}

La regulación antimonopolio evita que se produzca una concentración industrial de la actividad emprendedora de carácter internacional. Dicha concentración impide una sana competencia que incentive un desarrollo de la actividad emprendedora exportadora local. A su vez, la eficiencia de la legislación antimonopolio no se asocia al desarrollo de la actividad emprendedora de carácter internacional tendiente a la conformación de grupos económicos de peso económico.

Para el caso de México-Chile, la ineficiencia de la legislación antimonopolio permite la conformación de una actividad emprendedora de carácter internacional como se mostrará en la Figura 12. Sin embargo, en el caso de Perú-Colombia, la eficiencia de la legislación de la regulación antimonopolio ha servido para que la actividad emprendedora internacional de menor escala comercial se desarrolle y pueda incrementarse. Este efecto ambiguo de la eficiencia de la legislación antimonopolio se consolida a nivel del Acuerdo Comercial de la AP en un efecto negativo de 7.08 significativo al $1 \%$ como se mostrará más adelante en la Tabla 2. 


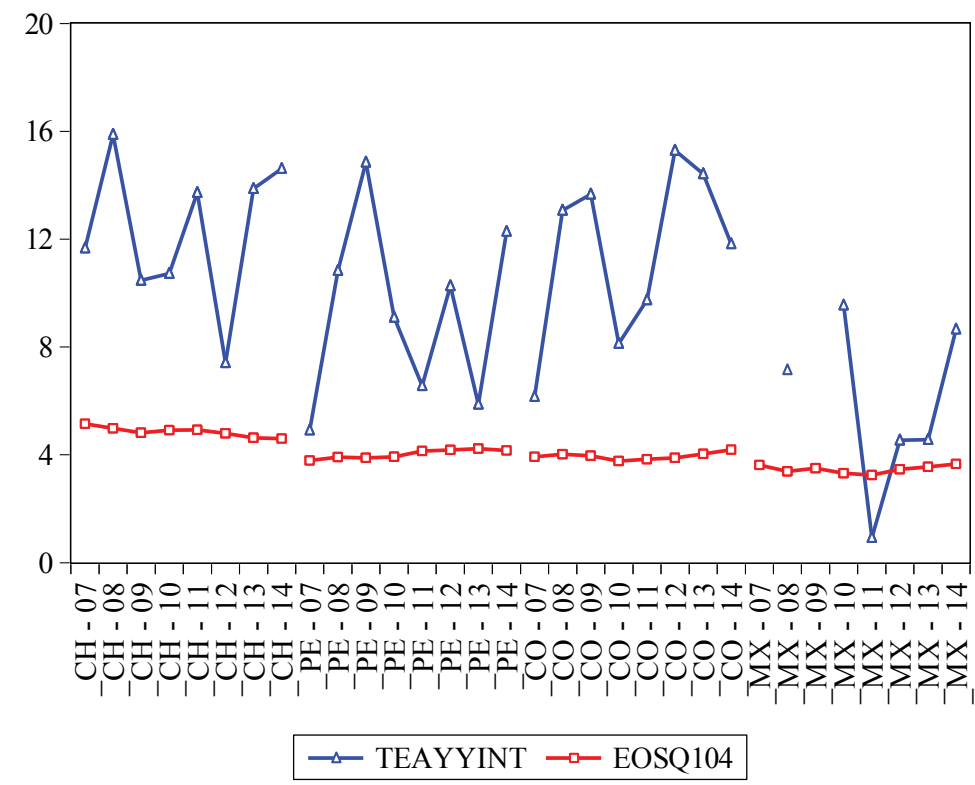

Figura 12. Regulación antimonopolio y actividad emprendedora internacional ( $\mathrm{CH}=$ Chile, $\mathrm{PE}=$ Perú, $\mathrm{CO}=$ Colombia, $\mathrm{MX}=$ México).

\subsection{Oportunidad de negocios}

La percepción de oportunidades de negocios, por parte del emprendedor asociado a la actividad emprendedora de carácter internacional, tiene una relación directa. En otras palabras, una percepción negativa, en torno a una oportunidad de negocios, induce a la reducción de la actividad emprendedora. Para el caso de Perú-Colombia, esta relación directa es estable, además de indicar que la actividad emprendedora en estos Estados parte implica una dependencia respecto a la percepción de oportunidad de negocios. Aunque, en el caso de México-Chile, esta relación es más fuerte por la profundización de la actividad emprendedora 
internacional y la importancia económica de las empresas emprendedoras de dichos países dentro del Acuerdo de la AP. Cuando la relación se observó a partir del Acuerdo de la AP, el peso menor de la actividad emprendedora internacional de los Estados parte, como Perú-Colombia, logró distorsionar la relación teórica esperada. Esto sucedió al punto de volverse negativa en 0.07 significativa al $1 \%$, lo cual se observará después en la Tabla 2. Esto se asocia con el grado de exposición al riesgo comercial y económico de la actividad emprendedora de los Estados parte como Perú-Colombia. Ahora bien, en la Figura 13 se ejemplificarán los datos aludidos.

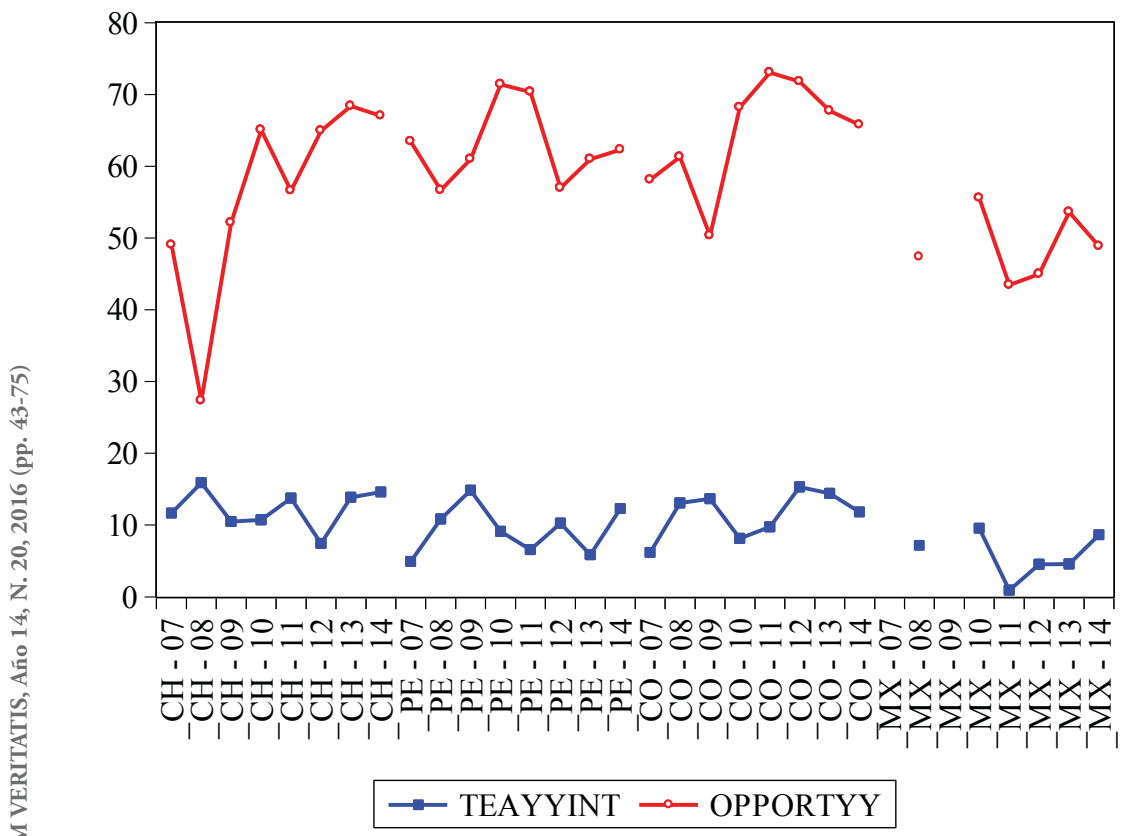

Figura 13. Oportunidad de negocio y actividad emprendedora internacional ( $\mathrm{CH}=$ Chile, $\mathrm{PE}=$ Perú, $\mathrm{CO}=$ Colombia, $\mathrm{MX}=$ México). 


\section{Resultados}

La presente investigación se ha realizado de acuerdo con el modelo relacional-teórico planteado. En primer lugar, se debe mencionar los factores de mayor peso para la actividad emprendedora en el contexto del Acuerdo Comercial de la AP, los cuales corresponden a factores objetivos macroeconómicos con la tasa de internacionalización empresarial como producto de la apertura económica que procede del Acuerdo. En segundo lugar, se encuentra la percepción subjetiva de los factores macroeconómicos que condicionan tanto la extensión de la actividad emprendedora internacional como la competitividad país desde el Estado parte de donde se genera la actividad emprendedora internacional. En tercer lugar, se encuentran las otras variables de análisis, que explican el comportamiento de la evolución de la tasa de actividad emprendedora internacional. Estos se mostrarán en la Tabla 2. 


\section{CÉSAR ANTONIO MONTERROSO CORONADO}

\section{Tabla 2}

\section{Modelo de Actividad Emprendedora Internacional}

Dependent Variable: TEAYYINT

Method: Pooled EGLS (Period weights)

Sample (adjusted): 20072014

Included observations: 23 after adjustments

Cross-sections included: 4

Total pool (balanced) observations: 92

Linear estimation after one-step weighting matrix

White diagonal standard errors \& covariance (d.f. corrected)

\begin{tabular}{crrrr}
\hline Variable & Coefficient & Std. Error & t-Statistic & Prob. \\
\hline C & -104.3074 & 15.67254 & -6.655424 & 0.0000 \\
D(VC4,1)/VC4 & 3.651135 & 0.415640 & 8.784376 & 0.0000 \\
IC & 16.71316 & 2.894045 & 5.775018 & 0.0000 \\
D(GI(-1),1)/GI(-1) & 3.510501 & 0.799892 & 4.388718 & 0.0000 \\
EOSQ068 & -3.264035 & 1.384786 & -2.357069 & 0.0209 \\
FGNMKTIDX & 14.34285 & 1.372894 & 10.44716 & 0.0000 \\
OPPORTYY & -0.074734 & 0.023462 & -3.185358 & 0.0021 \\
KNA11IMD & -2.216722 & 0.266164 & -8.328398 & 0.0000 \\
EOSQ118 & 22.89848 & 4.064425 & 5.633878 & 0.0000 \\
KNTCE & -0.007015 & 0.000942 & -7.448468 & 0.0000 \\
KNSBC & -0.588322 & 0.119292 & -4.931784 & 0.0000 \\
EOSQ353 & -9.137534 & 1.419738 & -6.436072 & 0.0000 \\
EOSQ104 & -7.081888 & 1.743368 & -4.062187 & 0.0001 \\
KNTF & 0.261491 & 0.115853 & 2.257103 & 0.0268 \\
\hline
\end{tabular}

Weighted Statistics

\begin{tabular}{lllr}
\hline R-squared & 0.966274 & Mean dependent var & 24.32839 \\
Adjusted R-squared & 0.960653 & S.D. dependent var & 27.29144 \\
S.E. of regression & 1.430692 & Sum squared resid & 159.6566 \\
F-statistic & 171.9021 & Durbin-Watson stat & 2.350720 \\
Prob(F-statistic) & 0.000000 & & \\
\hline & Unweighted Statistics & \\
\hline R-squared & 0.660328 & Mean dependent var & 11.32222 \\
Sum squared resid & 279.7499 & Durbin-Watson stat & 2.427494
\end{tabular}




\section{Conclusiones}

A partir de la investigación realizada, se puede alcanzar las siguientes afirmaciones relacionadas con las opciones de políticas públicas relacionadas con la actividad emprendedora internacional. Igualmente, se agregarán las propuestas y alcances de gestión del Acuerdo de la AP desde los factores objetivos y subjetivos relacionados empíricamente con la actividad emprendedora internacional:

- Para el desarrollo de una política pública de promoción de la actividad emprendedora internacional, el gestor debe tener muy presentes los factores ambientales al desarrollo de dicha actividad dentro del contexto del Protocolo Adicional de la AP. Igualmente, debe incorporar, en los objetivos de las Entidades de promoción del comercio en los Estados parte, los factores objetivos y subjetivos explicativos de la actividad emprendedora internacional; sin dejar de tener presente las heterogeneidades estructurales y sociales de los cuatro Estados partes como son México-Chile y Perú-Colombia.

- Para efectos de mejora del Protocolo Adicional en base a la implementación pendiente del tema de la integración regional para el fomento de la producción y consumo sustentable en el Grupo de Cooperación de la AP, el gestor de la política pública debe establecer determinados compromisos. Estos se deben dar en torno a la implementación de infraestructura portuaria que disminuya los costos y mejore la calidad de prestación de servicios a la exportación procedentes del Acuerdo Comercial de la AP. 
- El compromiso del Acuerdo Comercial de la AP, en los temas relacionados con la igualdad de oportunidades y el desarrollo de la innovación mediante el Comité de expertos, debe promover la búsqueda de una regulación antimonopolio de tratamiento uniforme entre los Estados parte.

- Un aporte de la investigación se relaciona con la clasificación de los factores objetivos propios de la operación de la actividad emprendedora y su grado de relevancia en la determinación de la política pública que el Grupo de Alto Nivel (GAN) presenta dentro del Acuerdo Comercial de la AP. Esto permitirá evaluar el grado de incidencia de las políticas públicas conformadas de acuerdo con la dirección y efecto cuantitativo observado de cada factor en la tasa de actividad emprendedora internacional.

- En el diseño de las políticas públicas de promoción y búsqueda de competitividad de la actividad emprendedora internacional, debe tenerse presente las evidencias empíricas de los factores subjetivos y ambientales. Ello se dará en la determinación de la relevancia de los factores objetivos en el grado de innovación empresarial mediante el gasto de inversión y desarrollo. Asimismo, estos últimos permitirán una evolución sustentable de la actividad emprendedora de carácter internacional en el contexto de la AP. 


\section{Referencias}

Bachetta, M., Beverelli, C., Cadot, O., Fugazza, M., Grether, J., Heible, M., Nicita, A., \& Piermartini, R. (2012). A Partical Guide to Trade Policy Analysis. New York, USA: United Nations.

García-Pérez de Lema, D., Gálvez-Albarracín, E., \& Maldonado-Guzmán, G. (2016). Efecto de la innovación en el crecimiento y el desempeño de las Mipymes de la Alianza del Pacífico. Un estudio empírico. Estudios Gerenciales, 32(141), 326-335.

Hors, I. (2015). How to foster the Internationalisation of SMEs through the Pacific Alliance Integration Process. OECD.

Molina, D., Heuser, C., \& Mesquita Moreira, M. (2016). Infraestructura $y$ desempeño de las exportaciones en la Alianza del Pacifico [Monografía]. Banco Interamericano de Desarrollo. Recuperado de https://publications.iadb.org/bitstream/handle/11319/7568/ Infraestructura-y-desempeno-de-las-exportaciones-en-la-Alianzadel-Pacifico.pdf

Reynolds, P., Bosma, N., Autio, E., Hunt, S., De Bono, N., \& Servias, I. (2005). Global Entrepreunership Monitor: Data Collection Design and Implementation 1998-2003. Small Business Economics, 24(3), 205-231.

Schwab, K. (2016). Insight Report The Global Competitiveness Report 20162017. Geneva, Switzerland: World Economic Forum. 